2013 to May 2018 were selected as subjects. NRS-2002 and BMI were used to assess the nutritional status of the entire group of patients, comparing the nutritional status of patients with Crohn's disease with intestinal fistula and non-intestinal fistula.

Results 66 patients with Crohn's disease had an NRS $\geq 3$ points in 134 cases; the incidence rate was $49.25 \%(66 / 134)$; $\mathrm{BMI}<18.5 \mathrm{~kg} / \mathrm{m}^{2}$ in 39 cases, the incidence rate was $29.10 \%$ (39/134). 19 patients with NRS $\geq 3$ points with non-intestinal fistula, the incidence rate was $28.78 \%(19 / 66)$; 9 patients with BMI $<18.5 \mathrm{~kg} / \mathrm{m}^{2}$ with intestinal fistula, the incidence rate was $23.07 \%$ (9/39). In patients with intestinal fistula, 47 patients had an NRS $\geq 3$, the incidence rate was $71.21 \%(47 /$ 66 ), and 30 patients with BMI $<18.5 \mathrm{~kg} / \mathrm{m}^{2}$, the incidence rate was $76.92 \%(30 / 39)$. The incidence of nutritional risk and malnutrition in patients with intestinal fistula was higher than that in patients with non-intestinal fistula (nutrition risk incidence: $71.21 \%$ vs $28.78 \%$; $<<0.05$; incidence of malnutrition: $76.92 \%$ vs $23.07 \%$; $<0.05$ ).

Conclusions Patients with Crohn's disease have a high incidence of nutritional risk and malnutrition. Patients with Crohn's disease and intestinal fistula have higher nutritional risk and malnutrition rates than patients with nonintestinal fistula. Therefore, nutritional support for patients with Crohn's disease during the perioperative period should be strengthened, especially in patients with intestinal fistula.

\section{IDDF2020-ABS-0054 CLINICAL STUDY ON THE CHANGES OF SERUM HCY AND PC IN PATIENTS WITH CROHN'S DISEASE}

Yi Yu*. Department of Critical Care Medicine, the Second Affiliated Hospital of Guangzhou University of Chinese Medicine, China

\subsection{6/gutjnl-2020-IDDF.56}

Background To investigate the changes of Hcy and PC in CD patients, and further explore the related factors of the prethrombotic state of CD patients.

Methods We collected clinical data of 65 patients with CD in our hospital from January 2012 to June 2016. 67 health examiners as controls. Fasting venous blood was collected in the morning. The serum Hcy and PC in CD patients were detected by ELISA method. The results of the test were compared with those in the control group, and prethrombotic state correlation analysis was also performed.

Results Compared with the control group, the serum Hcy in $\mathrm{CD}$ patients increased significantly $(6.78 \pm 2.17 \mathrm{ng} / \mathrm{ml}$ vs. $12.34 \pm 6.11 \mathrm{ng} / \mathrm{ml})$, and the difference was statistically significant $(\mathrm{P}<0.05)$, the serum $\mathrm{PC}$ in $\mathrm{CD}$ patients slight increased $(7.44 \pm 3.15 \mathrm{ng} / \mathrm{ml}$ vs $7.23 \pm 3.58 \mathrm{ng} / \mathrm{ml})$, while the difference was not statistically significant $(P>0.05)$. The correlation analysis showed that Hcy and PC levels in CD patients were positive correlate with prethrombotic state $(r=0.317$, $\mathrm{P}<0.05)$.

Conclusions The serum Hcy in CD patients was significantly higher than those in the control group. The correlation analysis showed that Hcy and PC levels in CD patients were positive correlate with prethrombotic state, these may due to the decrease of antithrombotic factors, and finally having a potential risk of thrombosis in $\mathrm{CD}$ patients.

\section{IDDF2020-ABS-0055 THE LONG-TERM OUTCOME OF ENDOSCOPIC RADIO INCISION WITH BALLOON DILATION OR WITH ESOPHAGEAL STENT PLACEMENT IN LONG-SEGMENT BENIGN ESOPHAGEAL STRICTURES}

Jui-yen Chen*, Jia-chuan Wu, Xiao-dong Chen, Li-fang Ye, Xiao-qiao Yang, Biao Liang Guangdong Second Provincial General Hospital, China

\subsection{6/gutjnl-2020-IDDF.57}

Background Endoscopic radio incision (ERI) is a novel treatment for benign esophageal strictures, especially for refractory stenoses. ERI provides a rapid improvement patency and exciting result in the short term. However, re-incision and/or dilation are required in some patients with long-segment strictures, and the long-term outcome of ERI is still controversial. The aim of this study was to evaluate the long-term efficacy of ERI with balloon dilation (BD) and ERI with esophageal stent placement (ESP) in long-segment benign esophageal strictures.

Methods This study was a randomized prospective trial. 37 patients with $1.5-5 \mathrm{~cm}$ length benign esophageal strictures from July 2015 to December 2018 were enrolled (the median age was 67 years old, range 49-74; male: female 25:12) and followed up for 12 months. All patients were randomly assigned to two groups: the ERI with balloon dilation group (ERI+BD, $\mathrm{n}=18)$ and ERI with esophageal stent placement group $(\mathrm{ERI}+\mathrm{ESP}, \mathrm{n}=19)$. The clinical data were recorded, such as the diameter of stenoses, length of strictures, dysphagia scores before and after the procedure, complications.

Results No severe adverse events were observed in either group. All patients in both groups were able to eat solid food within 1 week after the treatment. There was no significant difference between the ERI+BD group and the ERI+ESP group in dysphagia scores $(1.3 \pm 0.5$ vs $1.1 \pm 0.4, P>0.05)$ within 4 weeks after the procedure. In a 1-year follow-up, the relief rate of dysphagia symptom in the ERI+ESP group was higher than the ERI+BD group $(84.2 \%$ vs $44.4 \%, P=$ 0.029). Comparing to the ERI+BD group, the majority of patients in the ERI+ESP group could maintain lumen patency at 12 months $(78.9 \%$ vs $38.9 \%, P=0.032)$.

Conclusions Endoscopic radio incision (ERI) with esophageal stent placement (ESP) is the effective treatment for long-segment benign esophageal stricture, and provides a favorable result in the long-term.

\section{IDDF2020-ABS-0068 3D MODEL RECONSTRUCTION OF THE WHOLE STOMACH FROM STANDARD ENDOSCOPE VIDEO}

${ }^{1}$ Sho Suzuki*, ${ }^{2}$ Kenji Miki, ${ }^{1}$ Takuji Gotoda, ${ }^{3}$ Aji Resindra Widya, ${ }^{3}$ Yusuke Monno, ${ }^{3}$ Masatoshi Okutomi. 'Division of Gastroenterology and Hepatology, Department of Medicine, Nihon University School of Medicine, Japan; ${ }^{2}$ Department of Internal Medicine, Tsujinaka Hospital Kashiwanoha, Japan; ${ }^{3}$ Department of Systems and Control Engineering, School of Engineering, Tokyo Institute of Technology, Japan

\subsection{6/gutjnl-2020-IDDF.58}

Background Endoscopy is a common clinical practice to evaluate gastrointestinal diseases. Although endoscopy assesses gastrointestinal mucosal surface, it cannot evaluate 
the shape and volume of gastrointestinal organs and localization of the lesion. The accurate localization of a malignant lesion within the global view of the stomach is crucial for gastric surgeons to make a clinical decision of the operative procedure. Further, the shape of gastrointestinal organs possibly associates with some abdominal symptoms or disorders. Structure from Motion ( $\mathrm{SfM}$ ) is a method to recover $3 \mathrm{D}$ scene structure and camera motion from multiple images. SfM may be applied to endoscopy in order to reconstruct the shape of gastrointestinal organs. We aimed to reconstruct the $3 \mathrm{D}$ model of the whole stomach from standard endoscopic video image using SfM.

Methods Seven participants underwent gastroscopy under sedation for screening upper gastrointestinal diseases. The endoscope video was captured using a standard endoscope system. The video data was saved as an AVI format in 30 frames per second with full HD resolution. All endoscopic video was converted to RGB frames. The red channel of the extracted RGB frames were put to the SfM, where feature extraction, matching, pose estimation, and feature points triangulation were performed to generate a sparse $3 \mathrm{D}$ point cloud. Poisson surface reconstructed was performed to construct a 3D mesh model of the stomach. Finally, the texture of the endoscopic images was applied to the generated 3D mesh model to add more visual detail.

Results Most 3D point clouds were extracted from the red channel of the endoscopic images with the indigo carmine dye (figure 1). 3D meshes and texture representation resembling the whole shape of a stomach were generated from the cloud model (figure 2). Gastric ulcer lesion was clearly localized and reconstructed in one subject.

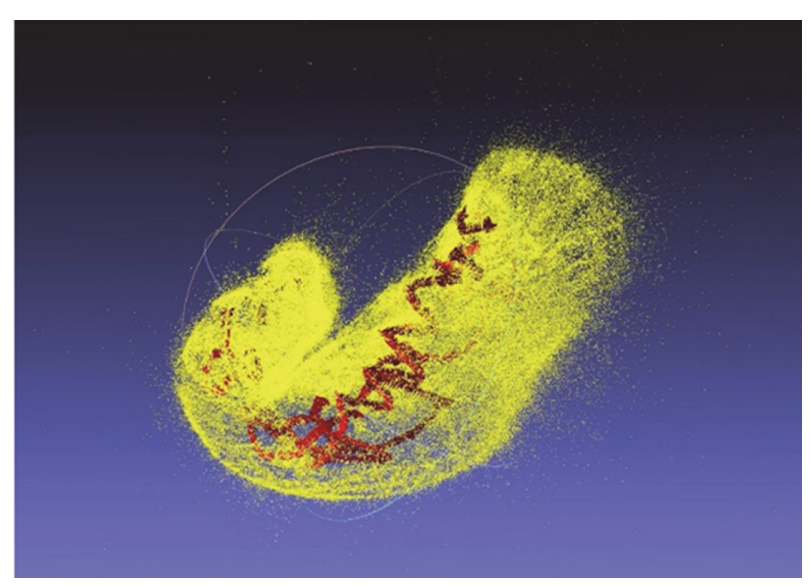

Abstract IDDF2020-ABS-0068 Figure 1
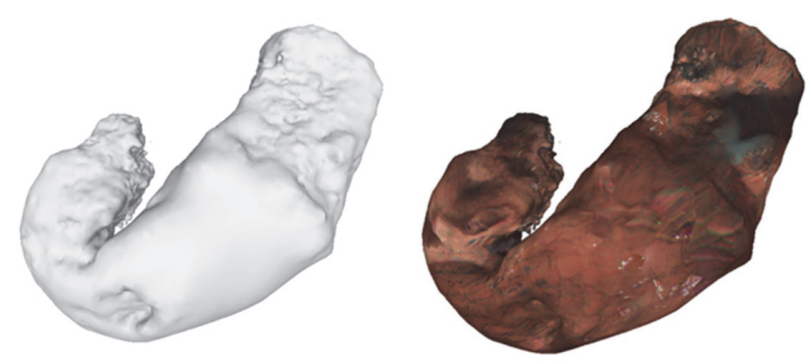

Abstract IDDF2020-ABS-0068 Figure 2
Conclusions Our study found that $3 \mathrm{D}$ reconstruction of the whole stomach can be achieved from a standard endoscopic video image using SfM. Furthermore, gastric ulcer lesion was also localized and reconstructed in $3 \mathrm{D}$ reconstruction model. Our future work will be focused on the clinical significance of our proposed method. We will try to evaluate the clinical usefulness of this method for the patients undergone surgery for early gastric cancers.

\section{IDDF2020-ABS-0070 PROTEOMIC ANALYSIS OF COLORECTAL CANCER WITH BRAFV600E MUTATION}

Biting Zhou*, Kailun Xu, Yingkuan Shao, Xi Zheng, Shu Zheng. Cancer Institute (Key Laboratory of Cancer Prevention and Intervention, China National Ministry of Education, Key Laboratory of Molecular Biology in Medical Sciences, China), The Second Affiliated Hospital, Zhejiang University School of Medicine, China

\subsection{6/gutjnl-2020-IDDF.59}

Background Proteomics provides new insights on tumorigenesis and progression of colorectal cancer (CRC) dependent of genomics and transcriptomics. BRAFV600E mutation is an unfavorable prognosis biomarker, occurring in about $5-20 \%$ CRC. In this study, we aimed to figure out the molecular characteristics in CRC with BRAFV600E mutation in proteomics.

Methods Based on a novel approach combining pressure cycling technology with the data-independent acquisition of mass spectrometry, thousands of peptides were obtained from formalin-fixed paraffin-embedded tumor tissue samples from 249 patients with colon cancer during 2009-2012 and then were processed through OpenSWATH to get the protein matrix. Differentially expressed proteins were analyzed by t-test ( $\mathrm{p}$-value $<0.05$, fold-change $>1.5 /<2 / 3$ ). BRAFV600E mutation status was tested by amplificationrefractory-mutation-system-based PCR assays. Biological function and pathway enrichment analysis were performed by STRING.

Results 27 patients were proved BRAFV600E mutation, indicating $10.8 \%$ occurrence rate in our CRC cohort. 276 differentially expressed proteins were obtained, including 243

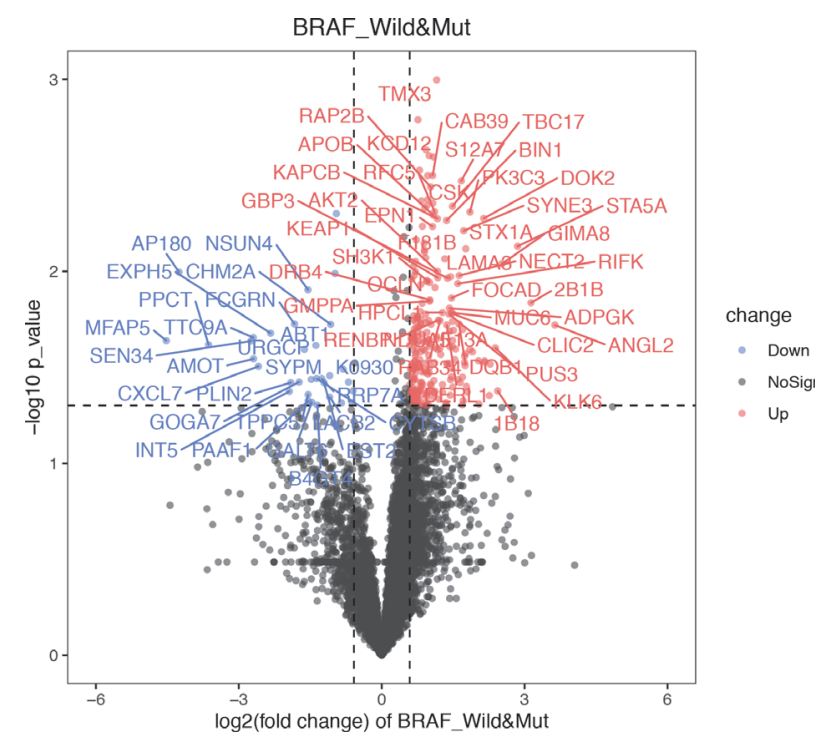

Abstract IDDF2020-ABS-0070 Figure 1 